\title{
Tourner en rond : le récit dans la gestion de crise en interaction de soin en réunion d'équipe ${ }_{1}$
}

\section{Going around in circles: Storytelling in crisis management in care interaction during team meetings}

\author{
Katja Ploog ${ }^{1}$ \\ ${ }^{1}$ Laboratoire Ligérien de Linguistique UMR 7270 CNRS, Université d'Orléans, France
}

\begin{abstract}
Résumé. L'évocation de la crise donne lieu, au sein de l'équipe médicale, à des élaborations discursives diverses et intriquées à travers l'articulation nécessaire de différentes temporalités dans l'élaboration du parcours de soin, allant de l'immédiateté de l'acte au processus de soin dans sa globalité. Nous proposons une réflexion sur les ressources mobilisées par les membres d'une équipe pluridisciplinaire en addictologie pour établir, au cours d'une interaction puis au fil des réunions, les contours de la crise, éléments de diagnostic qui détermineront ensuite l'ajustement du projet de soin ; le récit est une ressource majeure pour ce faire. L'étude exemplaire d'un ensemble d'extraits d'interactions de soin en réunion d'équipe (ISRE) consacrés à la crise d'un usager particulier pris en charge dans le service, nous permettra de dégager les caractéristiques du fonctionnement des récits dans ce contexte : la thématisation de l'état de crise de l'usager par l'équipe soignante "synchronise" les temporalités en alternant entre les énonciations situées (dans le présent actuel ou le passé) et les énonciations plus générales. Aussi, la progression thématique est souvent récursive.
\end{abstract}

\begin{abstract}
The mention of a crisis gives rise, within the medical team, to various and intertwined discursive developments via the necessary linking of different temporalities in the design of the care pathway, ranging from the immediacy of the act to the care process as a whole. We offer a study of the resources mobilized by the members of a multidisciplinary team in addiction therapy, to establish, in the course of an interaction and then in the context of several meetings, the outlines of the crisis, diagnostic elements which will then determine the adjustment of the care project; narratives are an important resource for this purpose. The exemplary study of a set of extracts of care interactions in team meetings (ISRE) devoted to the crisis of a particular user in care in the unit, will allow us to identify the characteristics of the functioning of the narratives in this setting: the thematization of the user's state of crisis by the care team "synchronizes" time frames by alternating between situated statements (in the actual present or the past) and more general ones. Consequently, the thematic progression is often recursive.
\end{abstract}

\footnotetext{
${ }^{1} \mathrm{Je}$ remercie tout particulièrement ma collègue Caroline Cance dont la contribution à ma réflexion sur l'interaction de soin est aussi constante que précieuse. Je remercie également mes relecteur/rice/s anonymes pour leurs commentaires et suggestions qui m'ont permis d'achever la rédaction présente de façon éclairée. Les erreurs et limites restantes n'engagent que ma propre responsabilité.
} 


\section{Crise et soin dans l'équipe pluridisciplinaire}

\subsection{La crise}

La crise peut être définie comme un «état instable qui, en l'absence d'intervention appropriée, se résout exceptionnellement de manière positive et évolue au contraire vers l'urgence $[. ..] »^{2}$ : aussi, la crise est appréhendée comme constellation interpersonnelle et, par là même, comme subjectivation d'un état exceptionnel, par le sujet en crise lui-même et par son environnement, comme une difficulté, voire comme une menace. L'apparition d'une crise résulte selon Formarier \& Jovic (2012 ; basé sur Kaës et al. 1979 et de Fondaire \& Larose 2007) d'un déséquilibre entre l'ampleur d'un problème donné, qui peut être géré par une adaptation particulière, et les ressources d'action disponibles pour y faire face. Si la tolérance des individus est variable face à la crise, la situation de vulnérabilité extrême (Chaudet 2020) atteint le noyau identitaire (Erikson 1982). De ce fait, la crise possède une dimension émotionnelle qui induit des comportements particuliers chez le sujet, qui peuvent, en conséquence, être perçus de l'extérieur. Nous retiendrons de la crise la manifestation d'une déstabilisation d'un système (a fortiori, d'une maladie), telle qu'elle est perçue.

Avec une méthodologie inspirée de l'analyse conversationnelle et de l'analyse de discours, il s'agira ici de mettre en exergue les ressources de l'élaboration discursive collective autour de la crise au sein d'une équipe soignante pluridisciplinaire en addictologie, qui s'emploie à travailler les ressources d'action pour permettre aux « soignés » d'affronter (mieux) les problèmes rencontrés.

Lors des réunions d'équipe, quelles sont les étapes de prise en charge de l'état de crise : comment celui-ci est-il repéré, caractérisé et "validé » collectivement ? Quelles sont les ressources qui permettent à l'équipe soignante de prendre en charge discursivement l'état de crise manifesté ? Comment la description évolue-t-elle au fil de l'interaction, et au fil de la crise ?

\subsection{L'équipe pluridisciplinaire}

Notre réflexion est fondée sur l'analyse qualitative d'interactions de soin issues du corpus « Parole émergée » (PE, Ploog, Equoy-Hutin \& Mariani-Rousset, 2014), recueilli dans un CSAPA (Centre de Soins, d'Accompagnement et de Prévention en Addictologie). Le centre offre aux personnes en situation d'addiction une prise en charge globale qui peut, selon la complexité des cas, s'étendre sur plusieurs années. Au-delà de la problématique médicale des traitements de substitution, ce terrain thématise un soin principalement non somatique dans l'idée du " care », fractionné sur des temps interactionnels successifs et réparti sur différents interlocuteurs médicaux et paramédicaux (médecin, infirmière, psychologue, éducateur), qui suppose en outre une coordination soutenue entre les membres de l'équipe.

Le corpus d'étude est composé de $9 \mathrm{~h} 30$ d'interactions de soin de type réunion d'équipe pluridisciplinaire (ISRE), dont l'objet est précisément la coordination et la remise en perspective filée des parcours de soin. Nous focaliserons notre analyse sur des séquences thématisant la crise d'un «usager ${ }^{3}$ »: c'est en effet ce terme qui est utilisé par les professionnels pour référer au public accueilli - terme ambivalent, mais de loin plus citoyen

\footnotetext{
${ }^{2}$ Circulaire $\mathrm{n}^{\circ}$ 39-92 DH PE/DGS $3 \mathrm{C}$ du 30 juillet 1992 relative à la prise en charge des urgences psychiatriques.

${ }^{3}$ Le corpus d'étude ne comporte en effet que des usagers hommes.
} 
et plus actif que ceux de soigné/e et de patient/e, et de ce fait plus en phase avec la Loi $2002^{4}$ qui définit le soin comme relation de partenariat entre différents acteurs coresponsables. Nous observerons comment la crise autour de l'usager est élaborée discursivement entre différentes temporalités (i.e. entre l'échelle de l'interaction et l'échelle du soin), pour développer le projet de soin tout au long du parcours. En particulier, nous mettrons en évidence le rôle central des récits qui donnent à voir la perception singulière de l'activité en lien avec une crise.

\title{
1.3 Le cas de David
}

Pour les besoins de l'exposé, notre propos se focalisera ici sur les échanges autour de la situation de David ${ }^{5}$, connu par le service depuis plus de 10 ans et repris en charge depuis quelques mois, après une interruption de plusieurs années. David se rend trois fois par semaine à l'unité méthadone, qui le suit de près et délivre son traitement. Dans une instabilité notoire, il oscille entre décrochages successifs et pratiques polyaddictes. Fort d'une expérience certaine avec toutes les structures d'aide sociale de la région, David sollicite de l'aide là où il y en a.

La personnalité compliquée de David rend le contact au quotidien difficile voire pénible pour les soignant/es, qui ont du mal à faire la part des choses entre mensonges et un certain déni de la situation par le concerné. Entre octobre et décembre, son cas est abordé collectivement à quatre reprises (R1, R2, R3, R4), en grande partie pour problématiser des objets externes au service d'addictologie, mais directement concernés par la prise en charge globale : d'abord, la stabilisation de la situation administrative de David est un vecteur central du projet de soin ; pour ne plus dépendre du CSAPA il lui faut une couverture sociale et ouvrir les droits à la Couverture Médicale Universelle. En réunion, l'équipe évalue la progression de la situation et remet en perspective les actions des professionnel/les pour accompagner David dans ses démarches. Sur fond d'agacement, l'équipe constate l'efficience toute relative des efforts déployés : « on tourne en rond », comme le formule le chef de service (sigle CHE, dans le corpus) dans la réunion R2. - Pas tout à fait, en réalité, car au final, la crise de David (et le processus interactionnel de sa prise en charge) révèle la fragilité du processus de soin et, peut-être, un état de crise de l'équipe soignante :

Réunion 2 (R2, durée 11'48, synthèse)

\begin{abstract}
Une partie de l'équipe est en congét. L'échange porte tout d'abord sur la demande de renouvellement de son allocation d'adulte handicapé. Il s'agit de déterminer qui sera le plus à même de renseigner le dossier : MED2 est en charge du suivi médical de David au sein du CSAPA, mais David ne respecte pas toujours les rendez-vous définis par le protocole. En parallèle, les modalités d'accompagnement par le médecin traitant questionnent l'équipe : David est soupçonné en effet de poursuivre les consommations tout en bénéficiant d'un traitement de substitution, en utilisant de façon abusive certains produits légaux pour lesquels son médecin traitant pourrait fournir
\end{abstract}

\footnotetext{
${ }^{4}$ Loi no2002-303 du 4 mars 2002 relative aux droits des malades et à la qualité du système de santé, JORF du 5 mars 2002, ELI : https://www.legifrance.gouv.fr/eli/loi/2002/3/4/MESX0100092L /jo/texte

${ }^{5}$ Usagers pseudonymisés.

${ }^{6}$ Locuteurs : INF1 et INF2 : infirmières : MED1 et MED2 : médecins addictologues (femmes), dont MED1 la responsable médicale du service ; CHE, chef de service (éducateur) ; PSY : psychologue, EDUC : éducateur.
} 
l'ordonnance. L'équipe décide de solliciter l'un des médecins internes à la structure (HERVE), et de ne confier le dossier à MED1 (ou MED2) que si HERVE n'intervient pas. L'échange thématise (de nouveau, mais cette fois-ci, indirectement) la dispersion du suivi de David sur de nombreux interlocuteurs, où personne n'a de vue complète. Ce qui peut sembler normal au vu du nombre de "chantiers" en cours, rend, dans le contexte, l'équipe méfiante vis-à-vis des informations obtenues auprès de l'usager. L'actualité médicale des soins dentaires urgents pour David fait glisser la discussion vers le problème du sous-encadrement médical dans le département. En cherchant une solution pour David, l'équipe formule de plus en plus explicitement ses difficultés dans la prise en charge non seulement de David mais aussi dans celle d'autres usagers.

Parmi les différents aspects de la trajectoire interactionnelle de ce processus, nous nous intéresserons en particulier aux attributs qui caractérisent l'usager et sa crise, et aux formats qui les caractérisent, dont, notamment, ceux en lien avec le récit.

\section{L'élaboration du projet de soin en interaction}

\subsection{L'interaction de soin en réunion d'équipe (ISRE)}

L'objet d'étude se focalise sur la réunion d'équipe : ce type d'interaction réunit tous les acteurs de l'équipe pluridisciplinaire de façon périodique, hebdomadaire dans le cas présent, pour évoquer ensemble l'actualité du service, dont un point sur les usagers entrants et sortants, des questions d'intendance, des requêtes particulières.

Une partie conséquente de la réunion est consacrée aux situations problématiques du moment. L'évocation des contours des crises a une place centrale dans la construction du projet de soin, dans un processus intersubjectif qui vise à mutualiser les informations et à identifier les ressources les mieux à même de répondre à la situation de la personne concernée. Notons que la participation active de l'usager à la construction du soin est importante, mais n'intervient que de façon indirecte dans notre étude des réunions d'équipe, qui se tiennent en l'absence de l'usager.

Une séquence d'ISRE consacrée à une situation individuelle se déroule donc schématiquement en trois étapes : (1) l'exposition des éléments concrets de la crise, (2) la généralisation des observations, éventuellement sous forme de diagnostic, et (3) l'explicitation d'un projet d'action en termes de prise en charge («intervention»). La séquence de la troisième réunion consacrée à David suit précisément cet ordre :

Réunion 3 (R3, durée 3'40, synthèse)

(1) L'infirmière explique que le dossier $\mathrm{MDPH}^{7}$ de David a été renvoyé avec une demande de complément.

(2) L'équipe s'interroge plus généralement sur les attendus de la MDPH, en citant les procédures en cours d'autres usagers.

(3) Sous l'impulsion du chef de service, l'équipe projette de renvoyer le dossier de David tel quel (pour respecter les délais) puis de contacter directement la MDPH par ailleurs.

\footnotetext{
${ }^{7}$ Maison Départementale des Personnes Handicapées.
} 
Ces étapes « macrostructurelles» caractéristiques de ce type de séquence peuvent être instanciées successivement, ou au contraire être intriquées, comme dans la première réunion :

Réunion 1 (R1, durée 3'02, synthèse)

\begin{abstract}
L'équipe met en commun les observations individuelles des membres sur l'état de santé dégradé de David (1). Prenant conscience du sérieux de la situation, le chef de service accepte de s'en occuper personnellement (3). L'infirmière insiste et esquisse la situation en supposant qu'il arrive au moment crucial du processus, celui où David doit être mis à contribution financière (2). Lorsque l'éducateur semble renouveler sa proposition d'aide pour orienter David sur un contrat de mutuelle à sa portée (3), l'infirmière argumente le caractère récurrent du comportement observé en exposant les démarches non abouties faites dans d'autres organismes (1), et en concluant sur le risque que les efforts consentis n'auront pour effet que l'abandon des soins par David (2).
\end{abstract}

Le constat initial (1) interroge la pertinence de la prise en charge actuelle, mais plusieurs interventions successives sont nécessaires pour faire émerger l'état de crise. En effet, la décision d'investir un nouvel interlocuteur dans l'accompagnement (3) ne semble pas une solution adéquate pour l'infirmière qui, dans cette interaction, ne parvient pas à se faire entendre malgré son insistance (2). Or, la volonté d'impulser une dynamique positive se heurte aux limites d'encadrement institutionnel dégradé (désert médical ; incohérences administratives) : derrière une crise en apparaît une autre.

\title{
2.2 Attributs
}

L'état conjoncturel de la crise tel que défini plus haut, est en réalité bien souvent itératif dans les contextes dont nous traitons. Sur fond de connaissances communes que l'équipe a pu accumuler au fil d'une expérience parfois très longue avec un usager et qui donne lieu à de nombreux implicites à un échange actuel, les membres de l'équipe doivent confronter leurs points de vue respectifs sur la crise du moment. Le travail d'explicitation mené peut se comparer à celui d'une " communauté interprétative » (Fish 2007), qui co-construit, dans différentes temporalités, le portrait de la personne en situation de crise. L'interaction en instance est interprétable au regard de la situation globale, qui intègre les caractéristiques du profil de la pathologie de l'usager, les modalités de fonctionnement du service de soin, ainsi que le contexte institutionnel et sociétal plus général, qui sont autant de facteurs qui auront un impact non seulement sur l'interaction elle-même mais aussi sur la mise en oeuvre d'un projet de soin cohérent, malgré tout.

L'élaboration de la crise dans l'interaction se fait par le procédé langagier général de la prédication d'attributs. Le processus rejoint dans une certaine mesure la problématique de la dénomination (Kleiber 1984) ou le paradigme désignationnel de Mortureux (1993), comme association située d'une structure linguistique à un référent extralinguistique, mais sans que celui-ci soit doté d'une stabilité / invariance : au contraire, la crise est l'une des dimensions du profil émergent de l'usager. Dans l'extrait Benzo/Codéine issu de la réunion 1 (résumé cidessus), la crise, explicitée par INF1 en terme de « il passe par quelque chose » (1.23), émerge parmi de nombreux attributs qui se suivent presque sans transition : 


\section{Benzo/Codéïne (R1, 0'32-1'27)}

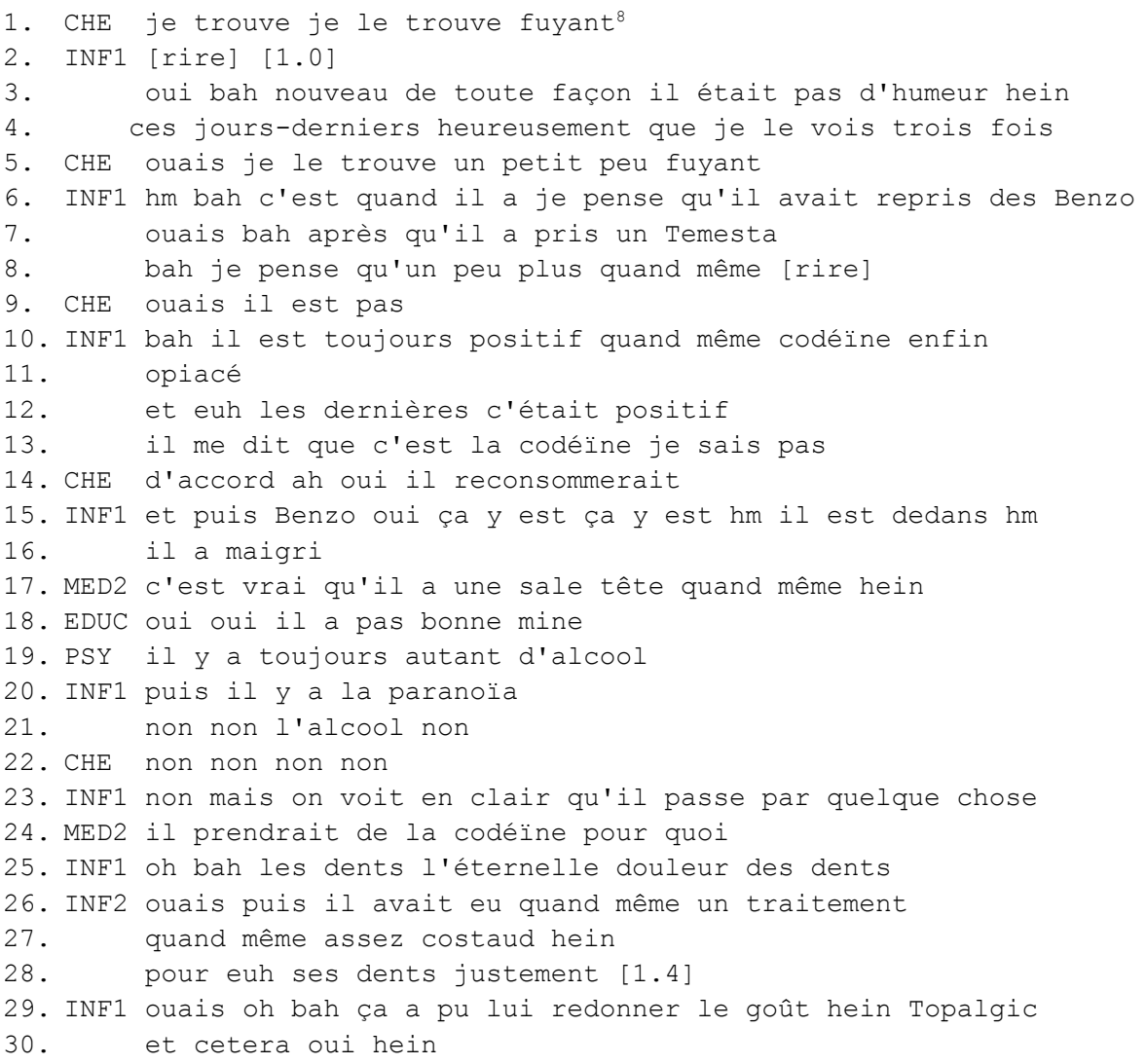

La construction des attributs dans l'espace interactionnel sous forme de liste peut s'expliquer dans le cas présent par la durée et l'intensité avec laquelle David fréquente la structure : David est identifié par tous, et chacun des membres peut contribuer activement à la coconstruction. Mais le nombre et la diversité des attributs caractérisent les ISRE de manière plus générale, où les différents acteurs mutualisent leurs informations et points de vue, et réfléchissent conjointement à la prise en charge la plus adaptée pour l'usager. Sur le plan sémantico-référentiel les attributs peuvent être décrits sur deux axes, à savoir, le type d'événement thématisé par la prédication et le caractère plus ou moins spécifique/particulier de l'attribut:

Tableau 1. Types d'attributs dans l'extrait Benzo/Codéine.

\begin{tabular}{|l|l|l|}
\hline $\begin{array}{l}\text { Benzo / } \\
\text { Codéïne (R1) }\end{array}$ & spécifique & générique \\
\hline \multirow{2}{*}{ procès } & $\begin{array}{l}\text { ouais bah après qu'il a pris un } \\
\text { Temesta bah je pense qu'un peu } \\
\text { il avait repris des Benzo }\end{array}$ & $\begin{array}{l}\text { il reconsommerait } \\
\text { (il'alcool) l'alcool non } \\
\text { (il prendrait de la codéine } \\
\text { pour quoi) les dents }\end{array}$ \\
\hline
\end{tabular}

${ }^{8}$ Conventions de transcription : crochets pour les éléments para- et non verbaux dont rires, silences (indiqués en secondes) ; [i] signale des segments verbaux non compris ; les parenthèses sont utilisées pour les commentaires ou informations complémentaires (interprétatives). 


\begin{tabular}{|c|c|c|}
\hline & $\begin{array}{l}\text { il me dit que c'est la codéine je } \\
\text { sais pas } \\
\text { il passe par quelque chose } \\
\text { il avait eu un traitement assez } \\
\text { costaud pour ses dents } \\
\text { ça a pu lui redonner le goût hein } \\
\text { Topalgic et cetera }\end{array}$ & $\begin{array}{l}\text { l'éternelle douleur des } \\
\text { dents }\end{array}$ \\
\hline & $\begin{array}{l}\text { il a maigri } \\
\text { enfin opiacé et euh les dernières } \\
\text { c'était positif }\end{array}$ & $\begin{array}{l}\text { je le trouve fuyant } \\
\text { je le trouve un petit peu } \\
\text { fuyant }\end{array}$ \\
\hline état & $\begin{array}{l}\text { il était pas d'humeur hein ces } \\
\text { jours derniers } \\
\text { il est toujours positif quand } \\
\text { même codéine }\end{array}$ & $\begin{array}{l}\text { ça y est il est dedans } \\
\text { il a une sale tête } \\
\text { il a pas bonne mine } \\
\text { il y a la paranoïa }\end{array}$ \\
\hline
\end{tabular}

Le type d'attribut est induit par des facteurs linguistiques tels l'aspect (grammatical, p.ex. le conditionnel dans « prendrait» ou « reconsommerait» et lexical, comme par le préfixe redans « redonner» ou " reconsommer ») et le temps verbal, ainsi que par la présence de modalisateurs verbaux et adverbiaux (" ça y est il est dedans », " toujours autant d'alcool »), et de nombreux d'indices multimodaux, dont des marques paraverbales (p.ex. rires, souffle), des indices vocaux (ex. timbre) et prosodiques tels l'accent, l'intonation, ou le débit; un aperçu des marqueurs multimodaux du discours est proposé par Ferré (2019). Une étude détaillée de la synchronisation des ressources multimodales et verbales pourrait montrer la complexité de cette élaboration sémantico-référentielle.

Retenons pour la suite de notre propos que la description des attributs sur les deux axes (activité, spécificité) se présente comme un continuum et que l'élaboration d'attributs nombreux et divers au fil de l'interaction donne lieu à des configurations variées. L'une des configurations majeures d'attributs, rendant compte des expériences personnelles, est le récit, ressource centrale de l'énonciation d'événements situés en dehors du hic et nunc et l'une des ressources majeures dans le développement conjoint : l'extrait Benzo/Codéine ci-dessus comporte en effet deux récits successifs, le premier rapportant la crise elle-même (1.1-18), à savoir, le fait que l'usager replonge dans la consommation, et le second les conditions d'émergence de celle-ci (1.23-30), à savoir, un traitement somatique connexe.

Notre contribution propose une étude exploratoire de la place du récit dans l'élaboration du projet de soin, en soulevant, à l'exemple de la situation de crise de David, quelques problèmes liés à la séquentialité interactionnelle dans laquelle ces récits émergent. Les séquences des quatre réunions d'équipe consacrées à David sont de durée inégale, et d'une hétérogénéité importante concernant les thématiques abordées, la configuration polylogale, etc. L'ensemble de ces quatre séquences boucle un cycle de crise, de sa première évocation à la probable sortie de crise. Le nombre de récits est ici donné à titre indicatif :

Tableau 2. Séquences ISRE commentées.

\begin{tabular}{|c|l|c|c|c|}
\hline séquence & \multicolumn{1}{|c|}{ Enjeu majeur } & date & durée & N récits \\
\hline R1 & D. replonge dans la consommation & 15.10 & 3 '05 & 5 \\
\hline R2 & Les « chantiers » n'avancent pas & 29.10 & $11^{\prime} 48$ & 16 \\
\hline R3 & Le dossier MDPH a été retoqué & 26.11 & 3 '36 & 3 \\
\hline R4 & D. s'installe dans son appartement & 10.12 & 1 '12 & 3 \\
\hline
\end{tabular}




\subsection{Le récit}

Qualifié de «big package» (Couper-Kuhlen \& Selting 2018, suivant Sacks 1992) dans la tradition interactionniste, le récit en interaction regroupe un éventail de pratiques de nature très diverse. Minimalement défini comme évocation d'évènements passés (Gülich \& Quasthoff 1986), ou, plus formellement, comme «a sequence of two clauses which are temporally ordered» (Özyıldırım, 2009), le récit a été étudié dans de nombreux contextes, par ses caractéristiques internes et les modalités de sa contextualisation.

Le modèle majeur de l'analyse des récits oraux d'expérience personnelle est celui de Labov \& Waletzky (1968), qui fait état de six étapes constitutives du récit: le résumé ; l'orientation; la complication; l'évaluation; la résolution; le coda. Les récits oraux d'expérience personnelle possèdent en effet des fonctions à la fois référentielle et évaluative. Si le modèle est basé principalement sur des récits élicités en situation d'entretien, la distinction de propositions (clauses) narratives et non narratives dans le modèle labovien implique la (possible) intrication du récit avec son environnement interactionnel. Pour le cadre du récit émergent en interaction, le modèle a été remis en perspective par Ochs (2014), qui identifie deux modalités narratives prototypiques, qui s'opposent dans cinq dimensions : le premier type repose sur un développement linéaire, par un narrateur principal, d'un ensemble d'événements d'intérêt notable ( « hautement racontable ») et moralement situés ; ce type de récit se démarque dans l'environnement interactionnel :

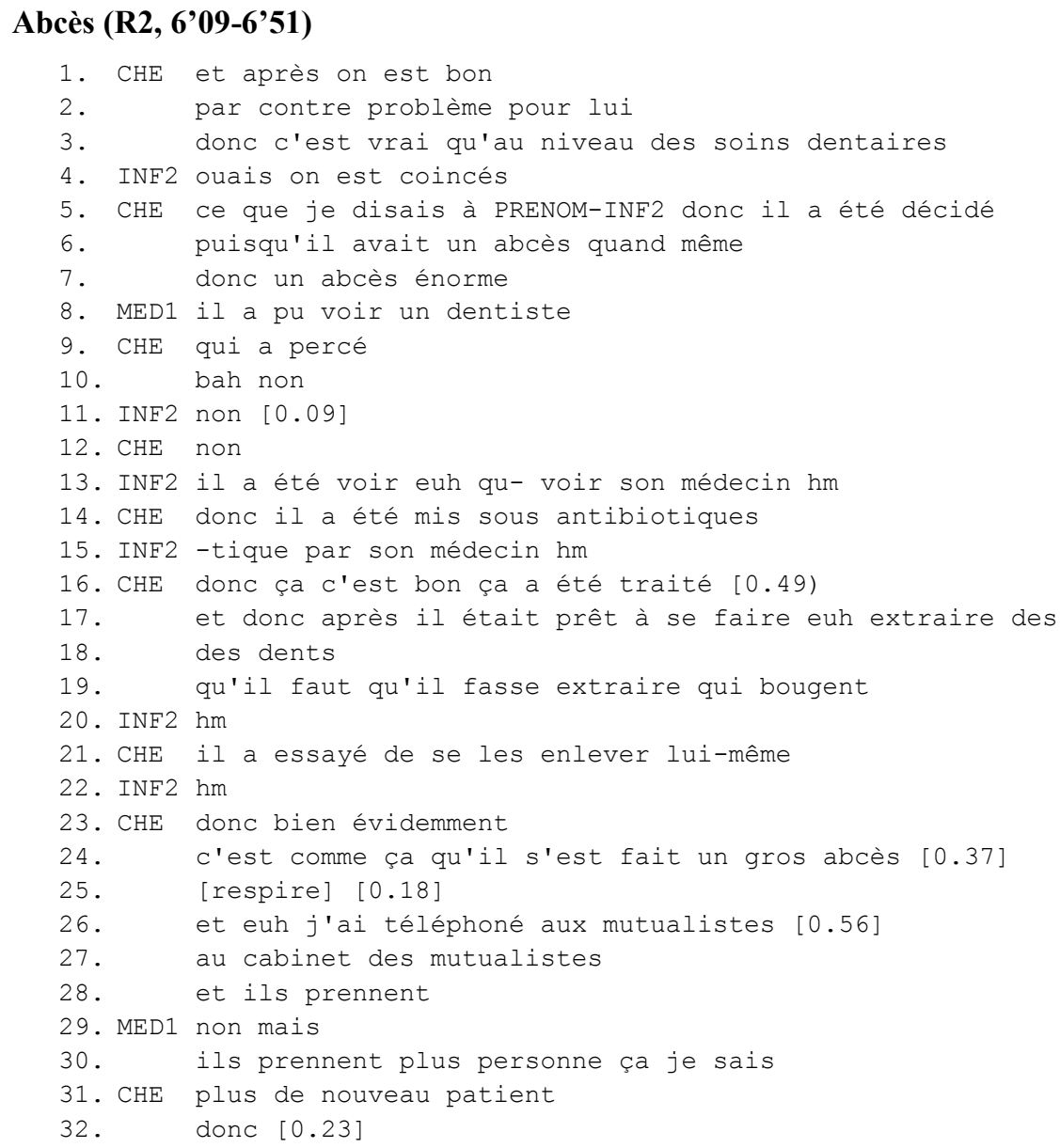




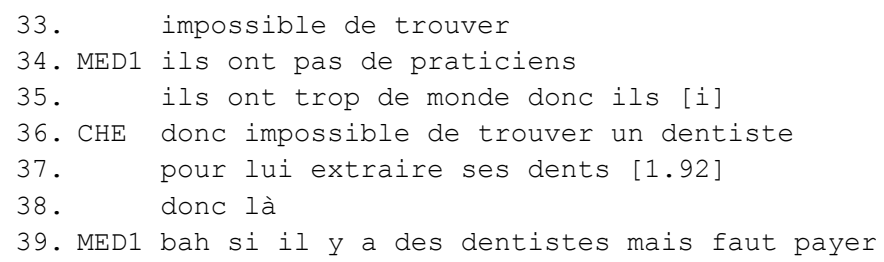

Ce récit est nettement distingué de l'interaction par un résumé initial (1.2/4: «par contre problème pour lui... »), un dénouement (1.36/37 : " donc impossible de trouver un dentiste pour lui extraire ses dents) et un silence prolongé, et, enfin, un coda (1.38 « donc là ») qui amorce le retour à l'échange. Le récit est élaboré par le chef de service (CHE), narrateur principal, qui associe explicitement l'infirmière (INF2) sur proposition de celle-ci (1.4), en la prenant à témoin (1.5).

Le second type de récit est celui d'une activité narrative ordinaire «qui invite les interlocuteurs à construire de manière dialogale des cadres au sein desquels organiser et interpréter des événements » (Ochs, 2014). Fruit d'un développement collectif, un tel récit élabore une expérience à faible intérêt, dont l'intrigue est floue (non linéaire) et l'évaluation morale fluctuante; il est fortement intriqué avec l'interaction dans laquelle il émerge. Mutuelle ci-après représente ce type de récit : il engage cinq membres de l'équipe : deux infirmières, l'éducateur et le chef de service, et une médecin ; l'intrigue Il faut accompagner David dans ses démarches pour l'obliger à aller jusqu'au bout est répartie sur trois pôles thématiques chacun co-élaboré par plusieurs acteurs : accompagner David (CHE, MED2, INF2); les démarches pour trouver une mutuelle économique (INF1, EDUC); David ne veut jamais payer (INF1, INF2).

\section{Mutuelle (R1, 1'36-1'59)}

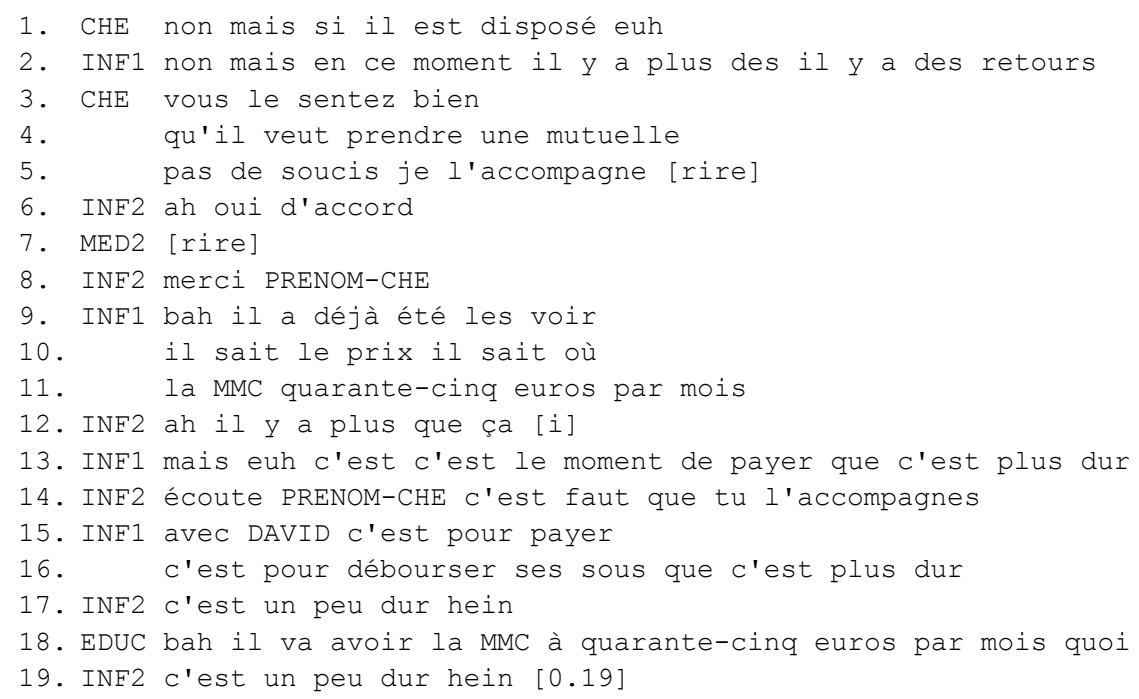

Les études des récits produits dans les interactions de soin ont porté généralement sur les récits de patients en santé mentale (Muntigl 2016 ; Turowetz 2015) ou dans le cadre de protocoles d'élicitation (Takagi 2019).

Comme nous l'avons illustré ci-dessus par les récits Abcès et Mutuelle, les deux (proto-)types dégagés par Ochs (2014) sont représentés dans le contexte interactionnel de la réunion de soin. Or, la plupart des récits analysés présentent des caractéristiques hybrides et ne se conforment pas à la macrostructure du modèle labovien. Les lignes suivantes permettront d'exposer quelques caractéristiques liées à la séquentialité des récits en ISRE. 


\section{Autour de la séquentialité}

\subsection{Séquentialité et temporalités}

Le plan temporel à plus petite échelle est l'énonciation d'attribut en temps « réel » d'interaction; il s'agit du niveau élémentaire de la construction discursive du soin par des fragments lexicaux et syntaxiques. L'échelle intermédiaire, l'interaction, comporte des séquences "thématiques » de construction du soin autour d'un usager donné, et des séquences à format spécifique comme les récits. L'échelle superordonnée est le parcours de soin, comportant au minimum une crise (celle qui conduit l'usager à se rapprocher de la structure de soin), qui s'étend sur une ou plusieurs ISRE et sera liée à des thématiques plus ou moins singulières pour l'équipe; toute énonciation d'attribut locale est rapportée au parcours de soin global.

Notre exposé permettra d'observer quelques aspects de la coordination de différentes séquentialités à l'échelle de la situation de crise, répartie ici sur quatre temps interactionnels dans un intervalle de deux mois. Ces quatre « réunions »- séquences d'interaction consacrées au même usager - R1, R2, R3, R4 comportent au total 27 récits, qui durent entre 6 et 64 secondes. La densité en récits est la moins importante dans la séquence la plus longue (R2), où seulement environ un tiers du temps est occupé par les récits ; dans les séquences courtes, la proportion dépasse les deux tiers. De même, les récits proportionnellement les plus longs sont situés dans les réunions plutôt courtes.

Dans le cadre des prises en charge de courte durée, l'activité de récit est généralement plus intensive en début de séquence. Aussi, plus la prise en charge de l'usager est récente et, en conséquence, l'expérience personnelle des soignant/es courte, plus la trajectoire interactionnelle suit la séquentialité standard des trois étapes (exposition des éléments de crise ; généralisation/diagnostic ; projection de la prise en charge). A contrario, l'évocation des prises en charge longues comme ici, typiquement liées aux cas compliqués avec des situations de crise récurrentes, présentent un déroulement souvent non linéaire des étapes (R1) et une intrication forte de l'activité de récit avec l'ensemble de l'interaction. Ici, les récits plus longs en début de crise sont entremêlés avec des attributs généraux (R1 : Benzo, présenté en 2.2). Un récit long en phase de sortie de crise semble en lien avec le bilan effectué : en R4, l'équipe dresse le bilan du parcours accompli au cours des derniers mois et projette l'étape suivante : la sortie de David du dispositif. Trois récits composent cet échange : le premier porte sur son installation dans l'appartement, le second sur la perspective de lui faire payer son traitement, et le troisième évoque les priorités d'accompagnement et les étapes accomplis. Atypique parmi les séquences de réunion, l'ensemble de cet échange très court est lui-même organisé comme un récit, sans trajectoire linéaire toutefois, mais en se terminant par un bilan : la sortie de crise paraît imminente.

\subsection{La séquentialité dans le récit}

Dans l'argumentation de Labov \& Waletzky (1968), la séquentialité chronologique est quasi «essentielle» du récit oral : du point de vue référentiel, le récit représente une séquence temporelle ${ }^{9}$, qui induit des formats syntaxiques précis (displacement sets, narrative heads) et conduit à un développement de l'intrigue ${ }^{10}$ comme trajectoire (i.e. marquée par les étapes début/milieu/fin). Le récit prototypique est donc instancié par un ensemble d'attributs

\footnotetext{
9 « one method of recapitulating past experience by matching a verbal sequence of clauses to the sequence of events which actually occurred », Labov \& Waletzky 1968.

${ }^{10}$ Labov n'emploie pas le terme d'intrigue (i.e. storyline), emprunté à Ricoeur (1983).
} 
spécifiques, typiquement ordonnancés de manière chronologique, comme dans le récit Abcès (R2) présenté ci-dessus en 2.3.

Le récit Codéïne ( $\mathrm{R} 1,23-30)$ présenté en 2.2, déclenché par la question de la médecin (MED2) de l'équipe, permet d'attribuer une explication au comportement de crise observé : le traitement des douleurs de dents par la codéïne a pu lui « redonner le goût» des produits psychoactifs et le faire replonger dans la consommation. Ce dernier aspect (la crise) est développé par le récit Benzo (R1) qui se trouve dans le contexte amont immédiat. L'on pourrait alors envisager de traiter comme un seul récit l'ensemble de la séquence Benzo/Codéïne, mais dont la linéarisation ne suit ni la séquentialité temporelle (qui serait douleur des dents $>$ traitement codéïne $>$ redonner le goût des produits $>$ surconsommation) ni causale (ordre inversé) des évènements, mais constitue un mélange des deux logiques.

Un récit comme Griller3 (R2), co-élaboré par MED1 et CHE, qui projette la séquentialité temporelle d'une démarche à suivre ne suit pas non plus l'ordonnancement chronologique des événements :

\section{Griller3 (R2, synthèse)}

Au cabinet $X$, un troisième dentiste vient d'arriver, il y a donc probablement des disponibilités pour des nouveaux patients. On devrait essayer de prendre RDV pour David, en présentant la situation particulière. Quand les collègues reviendront, on trouvera quelqu'un pour l'accompagner au rendez-vous. Sinon, il risque de ne pas y aller ou de se comporter mal, et on se fera griller.

\section{Griller3 (R2, 7'49-11'13)}

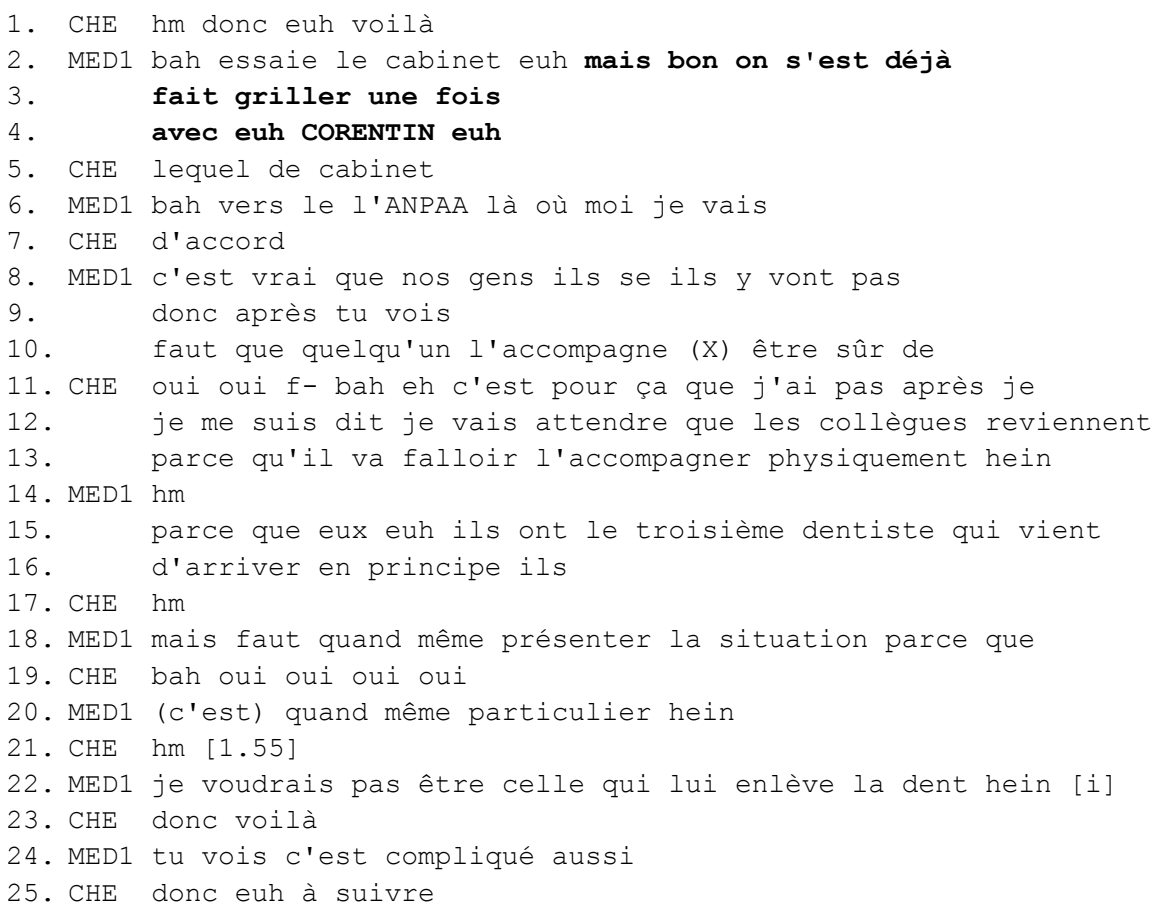

La séquence temporelle est virtuelle et son instanciation n'est pas linéaire : le risque de discréditer la structure en conséquence du désistement éventuel de l'usager est mise en avant, juste après la désignation du cabinet; la référence à la prise de rendez-vous précède l'information qui fait imaginer la vraisemblable disponibilité de ce cabinet ; enfin, l'option 
d'un accompagnement est formulée avant la condition opérationnelle, à savoir, le retour de vacances des collègues...

Beaucoup de récits analysés présentent des formats incomplets ou sont des récits «embryonnaires», en particulier, lorsque la séquentialité temporelle est réduite ou condensée :

\section{CMU1 (R2, 4'23-4'30)}

1. MED1 alors [0.61]

2. Iui ce qui aurait été bien [0.35]

3. je pense maintenant hein [0.43]

4. c'est qu'il y ait une AL un ALD(11) cent pour cent [1.41]

5. c'est ça qui aurait été bien [0.49]

6. et ça ça peut être fait que par un médecin généraliste

7. ou un médecin psychiatre

8. INF2 ouais

9. MED1 tu vois je suis en train de penser [0.21]

10. (puisqu', ce qu') on a galéré avec sa CMU [1.42]

11. tu vois

12. CHE ouais [0.20)

13. MED1 ça ç- ç- tu vois j'y pense euh [1.04]

14. CHE bah faudrait qu'on voie avec euh son médecin traitant

15. avec HERVE

Si le procès est explicité par une seule prédication (on a galéré avec sa CMU), il est toutefois représenté comme une trajectoire comportant différentes étapes, d'après le lexique verbal « galérer » longues et complexes, à ne pas en douter : s'agit-il d'un récit ? D'autres éléments démarquent la séquence de l'interaction : les indices prosodiques (silences), la présence d'une évaluation propre du procès contenu dans le terme, ratifiée par CHE, et le cadrage par les marqueurs métadiscursifs tu vois je suis en train de penser et ça tu vois j'y pense : il s'agit d'un évènement « hautement racontable» dans la typologie labovienne, car cela ne devrait pas se passer ainsi.

\section{Accompagner physiquement (R2, 9'36-9'42)}

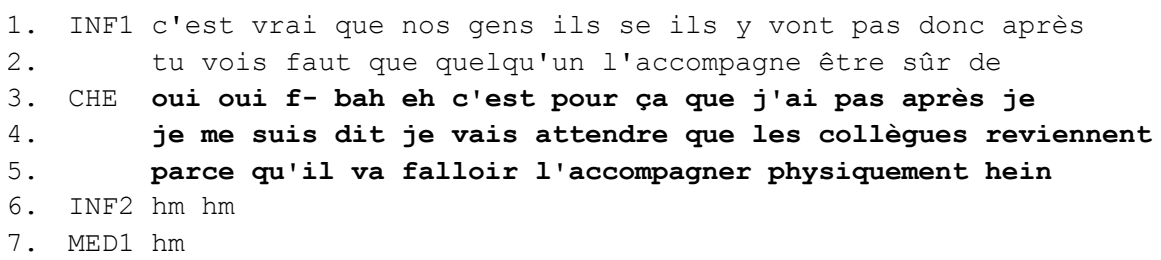

Ici, on note une amorce de clause ( $1.3:$ : $\mathrm{j}$ 'ai pas »), légèrement allongée, qui allude le fait que CHE n'a pas encore appelé ce cabinet (contexte amont), qui est suivie d'un marqueur de séquentialité («après) et du discours rapporté, autre format hautement caractéristique du récit (Rendulic 2018).

Tous les « récits » très courts, comme les deux qui ont été présentés, élaborent une expérience dotée d'une séquentialité certaine. En termes de produit textuel, il ne s'agit vraisemblablement pas d'une narration. Cependant, en terme d'activité, ces séquences participent de l'élaboration des mondes partagés, en rappelant les expériences communes ou déjà évoquées.

${ }^{11}$ ALD : Affection Longue Durée. 


\subsection{L'intégration du récit à l'interaction}

Contrairement à ce qui est mis en exergue par de nombreuses études, la « reconfiguration de la perception, construite au fil du temps, qu'[ont les narrateurs] de leur personne et du monde » (Ochs, 2014:52) n'est pas ici instrumentalisée à des fins communicatives, mais constitue la motivation même de l'élaboration du récit en ISRE : il s'agit, en effet, de dégager d'une expérience concrète un "diagnostic » partagé qui fournira la base d'un projet de soin commun. À cet égard, la dimension évaluative du récit, habituellement nécessaire pour l'interprétation de l'intrigue et donc de l'intérêt même de la narration (« Narratives are usually told in answer to some stimulus from outside, and to establish some point of personal interest », Labov et Waletzky 1968, figure 3), est intrinsèque aux récits produits en ISRE. La cohérence entre l'orientation et la complication s'articule ici entre l'usager, la thématique et la crise. Le récit, s'il est à propos, se justifie en tant que tel : il s'agit en effet d'une des particularités de l'ISRE que de thématiser les événements concrets dans le but même de les abstraire vers des catégories consacrées du soin.

La prise en charge collective de l'élaboration des récits par la « communauté de narration » (Ochs 2014) s'exerce sur tous les plans, séquentiel y compris. Nous ne pourrons pas nous attarder ici sur les rapports de place qui conditionnent le déroulement interactionnel. Mais la pluralité des narrateurs et la dynamique interactionnelle dans son ensemble favorisent une intégration forte des récits dans l'interaction.

Dans la plupart des récits étudiés, l'étape de l'orientation est très réduite, voire absente : en réalité, l'orientation superordonnée est l'introduction du nom de l'usager en début de (séquence de) réunion comme thème discursif. Dès lors, le récit émerge fréquemment d'une thématique saillante ou latente, ce qui peut expliquer la contiguïté ou l'imbrication de certains récits, qui prolongent une thématique précédemment élaborée. Le cas échéant, le résumé peut être donné sous forme de question ou de sollicitation d'un membre de l'équipe, qui peut être compris comme invitation à initier un récit. Certains récits sont amorcés par un attribut généralisant qui conduit à l'élaboration d'un récit dans la suite (Benzo, R2). De manière générale, le travail de formulation dans l'interaction conduit les locuteurs à faire des choix concernant le degré de précision de l'élaboration séquentielle. De ce fait, beaucoup de récits comportent des attributs généralisants, qui se combinent aux éléments particularisants ou qui sont ré-élaborées par un récit plus spécifique dans le contexte amont ou aval (Grillerl et Griller2, R2).

L'évocation en ISRE d'un événement particulier extérieur à cette interaction lui confère une dimension évaluative certaine : le récit sert à argumenter en quoi la crise en est une. En cela, nous avons affaire à des expériences « hautement racontables », et nos récits se démarquent pour la plupart par des postures morales généralement assez certaines, sans qu'elles soient nécessairement consensuelles. Par exemple, à la fin d'Abcès (présenté en 2.3), la MED1 s'impatiente du rythme de progression et anticipe ce qu'elle imagine être la résolution (1.30) et qu'elle va contester dans la suite (1.39). L'issue du récit montre donc l'appréciation différente de la situation par CHE et MED1, qui s'apprêtent à engager des trajectoires différentes pour la suite de l'interaction.

Le dernier récit de $\mathrm{R} 4$, qui semble faire le bilan du parcours accompli, est particulièrement polyphonique. Le récit est déclenché par la perspective de la sortie de David du dispositif :

\section{Vous ne faites rien pour moi (R4, 0'31-1'05)}

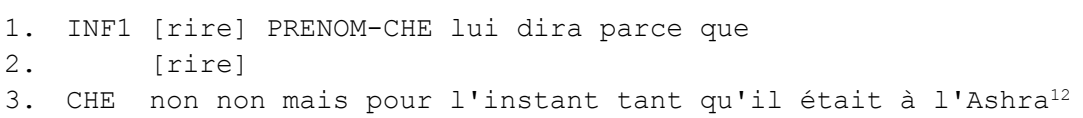

\footnotetext{
12 Centre d'accueil de nuit.
} 


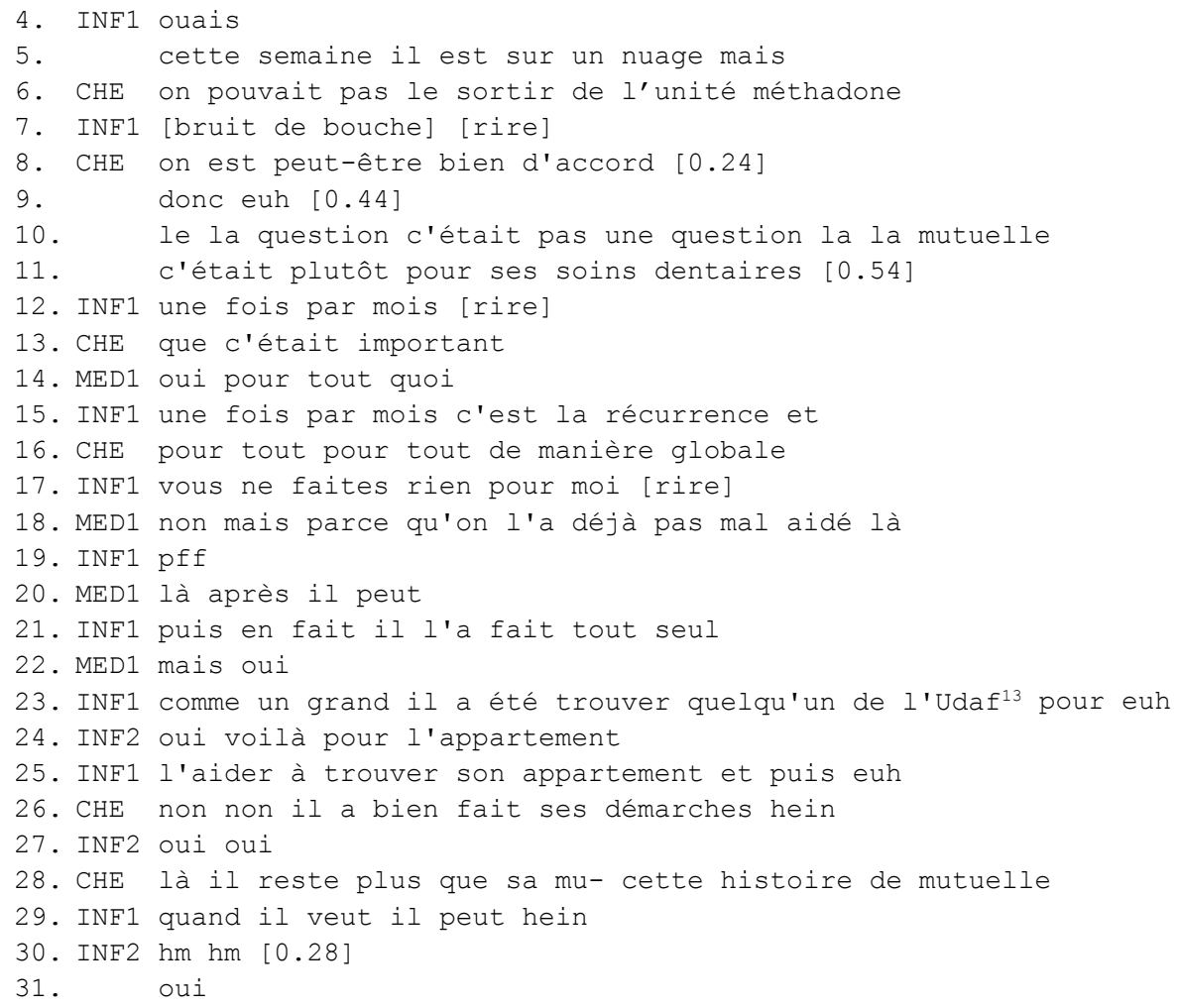

Instancié conjointement / parallèlement par INF1 et CHE (en plus des contributions de INF2 et MED1), et partant du moment présent (transition entre la vie en foyer et en appartement), le déroulement global comporte deux trajectoires très différentes :

- INF1 rappelle les plaintes récurrentes de David de ne pas recevoir suffisamment de soutien (un discours rapporté avec une voix théâtralisée, 1.17), alors qu'il semble pouvoir se prendre en charge $(1.23,1.29)$.

- Étant sollicité par INF1 pour annoncer la nouvelle à David, CHE rappelle les conditions du maintien prolongé : l'étape de l'appartement était une condition nécessaire $^{14}$, alors que la contraction de la mutuelle était urgente pour les frais dentaires ; CHE semble vouloir terminer cette étape avant la mise en relais.

Ces deux trajectoires caractérisent des récits à part entière, mais liés par la communauté d'interprétation : dans les deux récits, on entend la lassitude de INF1, qui se trouve au contact quotidien avec David et qui ne souhaite pas lui annoncer la nouvelle de la mise en relais, craignant sa réaction. De la perception de la complexité de la situation et des obstacles auxquels se confronte le soin, le développement discursif dépeint parfois une crise qui engage tout autant les soignant/es que le/la soigné/e, et qui est alors une crise globale du soin.

\subsection{Une progression thématique récursive}

La formulation d'événements à caractère spécifique tels qu'ils sont explicités par un récit contribuent à élaborer le profil du soigné par des attributs concrets (situés), pour pouvoir

\footnotetext{
${ }^{13}$ Union départementale des associations familiales.

${ }^{14}$ Confier le traitement de substitution à un usager hébergé en foyer pose de nombreux problèmes liés au stockage et à la sécurité.
} 
progressivement prendre la distance nécessaire et aboutir à un relatif consensus indispensable pour la construction d'un projet de soin cohérent. Or, dans bien des cas, nous observons une récursivité dans l'émergence des récits. Cinq thématiques sont élaborées par plusieurs récits au fil des réunions; les trajectoires esquissées sont diverses. La redondance sémanticoréférentielle en plus des symétries lexicales placent sans doute certains récits dans un rapport de reformulation ou d'étayage. Mais bien souvent, ces ré-élaborations ont une fonction argumentative dans l'interaction, en particulier, de travailler l'évaluation de la situation de crise. Autrement dit ces récits contribuent à orienter le projet d'action en cours d'élaboration. Ces caractéristiques donnent à voir le projet de soin émergent, fragile et questionnable. On observe enfin des variations d'échelle, en alternant entre l'expérience concrète et la synthèse plus générale, qui font résonner le récit avec le parcours de soin dans son ensemble.

Par exemple, le thème de la CMU est élaboré à trois reprises :

CMU1 (R2, 4'23-4'30) : présenté en 3.2

\section{CMU2 (R2, 5'12-5'28)}

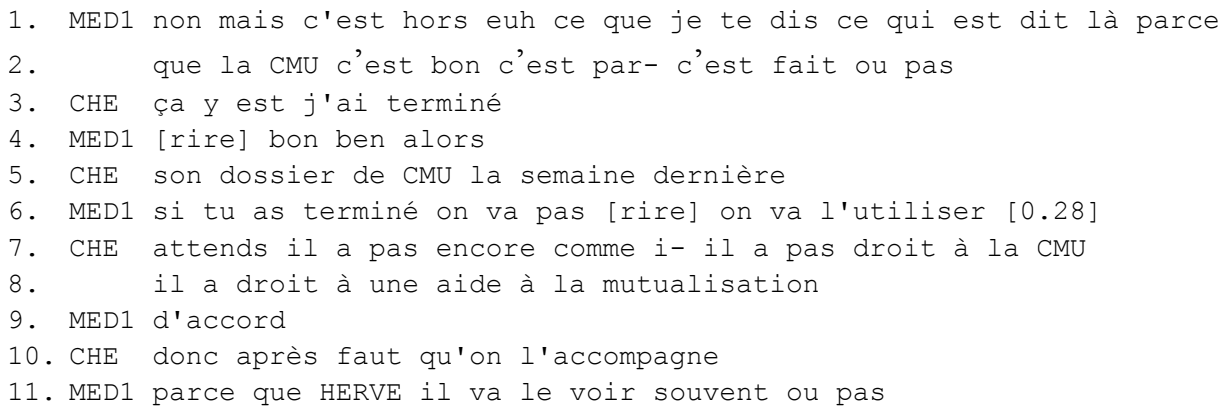

\section{CMU3 (R2, 5',41-6'11)}

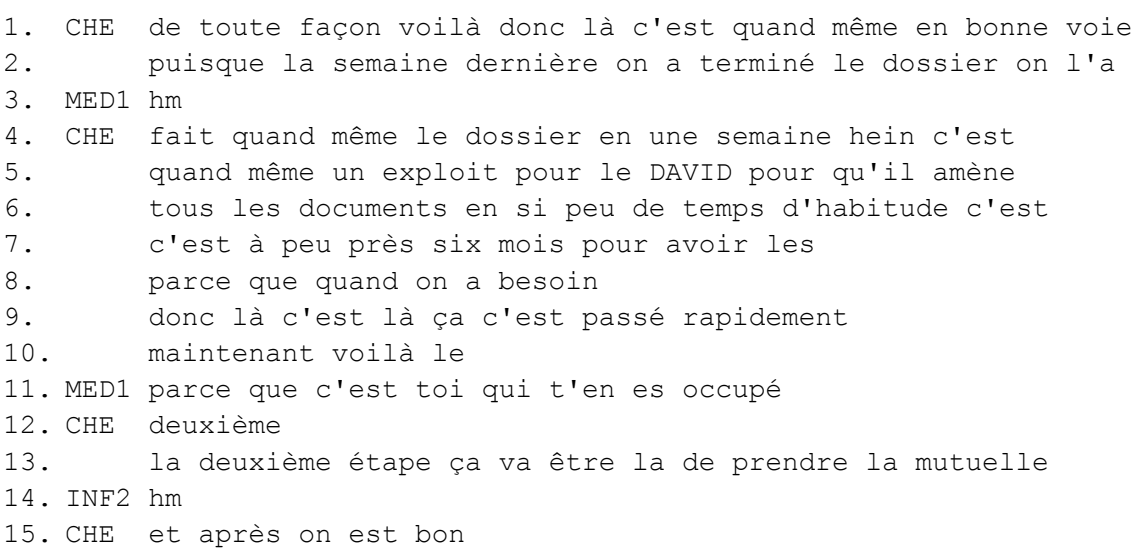

Ici, la fonction d'étayage est patente : minimalement développé en CMU1, le thème est repris sous forme de microrécit en CMU2 (1.3-6), puis développé dans une séquentialité temporelle plus typique dans CMU3. L'évaluation de CMU3 (ça s'est passé rapidement, 1.9) s'inscrit d'ailleurs en faux avec celle de CMU1 ( on a galéré »).

Dans R2, on trouve aussi le récit Bon moment (10’09-10’16) qui reprend Abcès (6’09-6’51) en appuyant légèrement l'évaluation : 


\section{Bon moment (R2, 10'09-10'16)}

1. CHE mais c'est vrai que là c'était le bon moment de le traiter

2. puisqu'il y avait plus d'abcès il y avait voilà c'était

3. idéal mais le problème c'est qu'on trouve pas de praticien

À l'échelle de l'interaction, cette nouvelle élaboration correspond au cadrage (par le chef de service) relatif à un problème non résolu. Le besoin d'insister pour mieux faire entendre son point de vue caractérise aussi la série des trois récits Griller : les deux premiers se suivent de près, le premier étant d'ordre général (MED1), le second plus concret (INF2) :

\section{Griller1/Griller2 (R2, 7'48-8'13)}

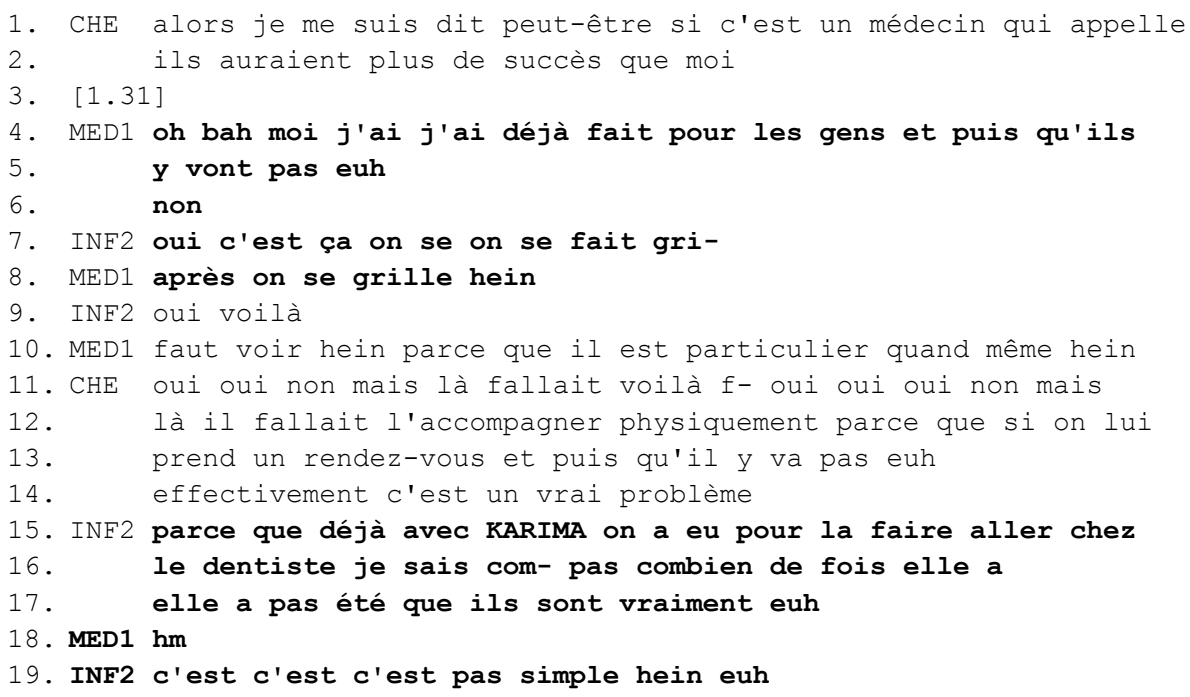

Griller3 (présenté en 3.2) est à nouveau initié par MED1, mais qui élabore cette fois-ci elle aussi un cas concret, en guise d'argument supplémentaire.

L'accompagnement, qui donne lieu à sept récits, est la thématique la plus élaborée. Ceci s'explique en partie par la centralité de cette mission pour le service : les quatre récits en R2 détaillent, de façon partiellement redondante, les démarches déjà effectuées ( 2 récits) et celles projetées ( 2 autres récits) à l'échelle de l'interaction; celui en R1 rapporte les accompagnements inaboutis dans d'autres organismes ; celui en R3 le projet d'action du moment (échelle de l'interaction) ; celui en R4 revient sur l'ensemble de la prise en charge, à l'échelle du soin.

Enfin, la mise à contribution financière de David est élaborée par quatre récits (en R1, R2, R4). Tout au long de la crise, l'évaluation semble presque stable... Ce sont surtout les ressources qui évoluent : dans Mutuelle (R1, présenté en 2.3), la difficulté de faire payer David est énoncée explicitement et de façon très redondante à propos d'un événement concret ; dans Payer 1, MED1 reprend ce thème en généralisant (« il ne peut jamais payer »), et dans Payer 3, le rythme de progression de l'interaction crée un quiproquo qui est accueilli dans l'hilarité générale :

Payer3 (R4, 0'22-0'27)

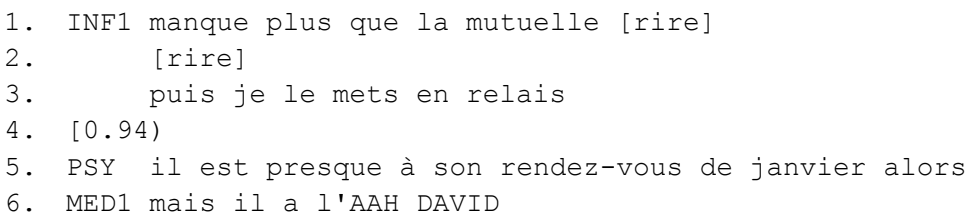




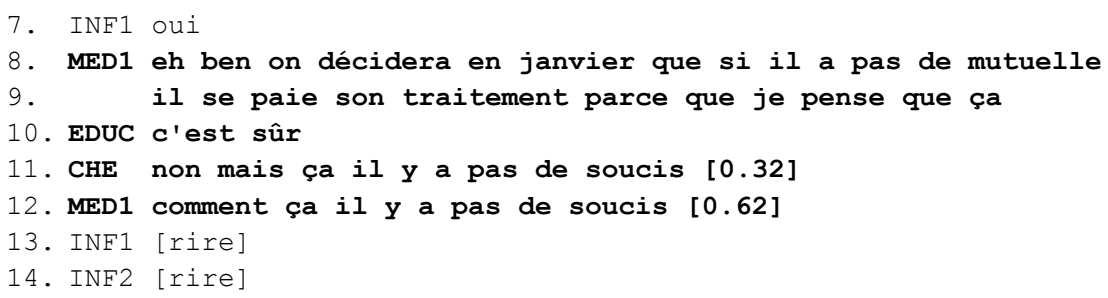

Après avoir avancé en R2 que David serait prêt à payer sa mutuelle (Payer2), CHE se montre à nouveau optimiste ici au sujet de l'issue du processus engagé...

\section{Récit et élaboration discursive de la crise}

Dans les réunions d'équipe en contexte de soin, l'interprétation collaborative des événements est une méthode majeure pour la définition du projet de soin. Le récit prend place naturellement dans cette dynamique interprétative. Nous avons montré comment les récits dans les ISRE permettent de co-articuler différents attributs qui élaborent le profil de l'usager au fil de la remise en perspective du parcours de soin, le cas échéant, au fil des crises. Certaines caractéristiques dégagées font apparaître les formats de récits comme non canoniques: la dynamique interactionnelle orientée du passé (expérience contée) vers l'avenir (le projet de soin) induit une diversification des ancrages temporels (passé, présent, conditionnel, futur) ; les récits sont mêlés à des attributs génériques, et les récits eux-mêmes peuvent condenser des expériences spécifiques en terme de généralisations et rendent incertaine la limite entre description et narration ; au regard de l'élaboration variable de la densité/homogénéité d'un événement, la trame séquentielle du récit est une trajectoire temporelle élaborée autour d'un ou plusieurs événements spécifiques, où les étapes du processus (début/milieu/fin) ne sont pas toujours explicitées.

La structuration linéaire où le récit peut être entrecoupé d'autres attributs qui ne relèvent pas du récit semble directement induit par le contexte interactionnel, où la finalité de l'échange est de converger vers un projet, commun : indirectement, la donnée co-constructionnelle (i.e. la communauté d'interprétation) conduit à l'émergence d'un macro-format qu'on pourrait qualifier de "récit filé ", mêlé à l'activité interactionnelle, combinant différentes temporalités, et qui n'est pas toujours clairement délimité.

Le présent énonciatif dans lequel se construit le récit permet en effet de faire la jonction entre la crise (le déséquilibre) observé et les actes susceptibles de la dépasser (le soin). Le récit apparaît donc comme une ressource centrale dans l'explicitation du constat de crise et permet, dans la temporalité supérieure, dans la projection du prolongement du processus du soin.

\section{Références}

Bres Jacques. (1994). Le récit oral suivi de Questions de narrativité, Montpellier, Praxiling. Chaudet Vincent. (2020). Parler et penser en équipe l'admission et l'accompagnement éducatif d'enfants placés au regard de leur situation extrême, Éducation et socialisation 57 (URL : http://journals.openedition.org/edso/12433; consulté le 21 avril 2021).

Couper-Kuhlen Elizabeth et Selting Marget. (2018). Interactional Linguistics: Studying Language in Social Interaction. Cambridge: Cambridge University Press.

Drew Paul et Heritage John. (1992). Analyzing Talk at Work : An Introduction, in P. Drew et J. in P. Drew / J. Heritage (eds), Talk at work, Cambridge: Cambridge University Press, p. 3-65. 
Erikson Erik H. (1982). Enfance et société. Paris : Delachaux et Niestlé.

Fish Stanley. (2007). Quand lire c'est faire. L'autorité des communautés interprétatives, trad. DDe l'américain par E. Dobenesque, Paris, Éd. Les Prairies ordinaires.

Fondaire Marie et Larose Suzanne. (2007). Détresse psychologique en situation de crise. Ed Quebecor, Montréal.

Formarier Monique et Jovic Ljiljana. (2012). Les concepts en sciences infirmières: 2ème édition. Toulouse, France: Association de Recherche en Soins Infirmiers.

Gülich Elisabeth et Quasthoff Uta. (1986). Story-telling in conversation: Cognitive and interactive aspects. Poetics. Elsevier 15(1-2). p. 217-241.

Kaës René, Anzieu Didier et Missenard André. (1979). Crise, rupture et dépassement psychologique. Ed Dunod.

Kleiber Georges. (1984). Dénomination et relations dénominatives, Langages, 76, p. 77-94.

Labov William et Waletzky Joshua. (1968). Narrative Analysis, in W. Labov et al. (éds.), $A$ Study of the Non-Standard English of Negro and Puerto Rican Speakers in New York City (pp. 286-338). New York : Columbia University Press.

Mortureux Marie-Françoise. (1993). Paradigmes désignationnels, Semen 8, p. 123-141.

Ochs Elinor. (2014). Ce que les récits nous apprennent, Semen 37 (URL : http://journals.openedition.org/semen/9865 ; consulté le 04 mai 2021)

Muntigl Peter. (2016). Storytelling, depression, and psychotherapy. In Jessica Nina Lester \& Michelle O'Reilly (eds.), The Palgrave Handbook of Adult Mental Health, London: Palgrave Macmillan, p. 577-596.

Özyıldırım Işı1. (2009). Narrative analysis: An analysis of oral and written strategies inpersonal experience narratives, Journal of Pragmatics 41, p. 1209-1222.

Ploog Katja, Equoy-Hutin Séverine et Mariani-Rousset Sophie. (2014). Parole émergée. Corpus d'interactions de soin en structure pluridisciplinaire. Besançon : Université de Franche-Comté. Métadonnées publiées sur https://cocoon.huma-num.fr/exist/crdo.

Ploog Katja, Mariani-Rousset Sophie et Equoy-Hutin Séverine (eds.). (2018). Emmêler et démêler la parole. Approche :pluridisciplinaire de la relation de soin. Annales Littéraires de l'Université de Franche-Comté (série Linguistique-SémiotiqueCommunication).

Rendulic Nina. (2018). Discours représentés et transformation de la réalité dans les récits oraux, in H. Barthelmebs-Raguin/G. Komur-Thilloy/ J.M. Lopez-Muñoz, S. Marnette \& L. Rosier (eds.), Le Discours rapporté. Temporalité, histoire, mémoire et patrimoine discursif, Paris: Classique Garnier, p. 287-301.

Ricoeur Paul. (1983). Temps et récit (tome 1). Paris : Ed.Seuil.

Sacks Harvey. (1992). Lectures on Conversation. Oxford : Blackwell.

Turowetz Jason. (2015). The interactional production of a clinical fact in a case of autism. Qualitative Sociology 38(1), p. 57-78.

Takagi Tomoyo. (2019). Referring to past actions in caregiver-child interaction in Japanese. Research on Children and Social Interaction 3(1-2), p. 92-118. 